University of Wollongong

Research Online

Australian Institute for Innovative Materials -

Papers

Australian Institute for Innovative Materials

$1-1-2016$

Characterisation of graphene fibres and graphene coated fibres using capacitively coupled contactless conductivity detector

Joan M. Cabot

University of Tasmania

Emer Duffy

University of Tasmania

Sinead Currivan

University of Tasmania

Andres Ruland Palaia

University of Wollongong, ruland@uow.edu.au

Rouhollah Jalili

University of Wollongong, rjalili@uow.edu.au

See next page for additional authors

Follow this and additional works at: https://ro.uow.edu.au/aiimpapers

Part of the Engineering Commons, and the Physical Sciences and Mathematics Commons

Research Online is the open access institutional repository for the University of Wollongong. For further information contact the UOW Library: research-pubs@uow.edu.au 


\title{
Characterisation of graphene fibres and graphene coated fibres using capacitively coupled contactless conductivity detector
}

\begin{abstract}
The use of capacitively coupled contactless conductivity detection (C 4 D) for the characterisation of thin conductive graphene $\mathrm{fi}$ bres, graphene composite $\mathrm{fi}$ bres, and graphene coated $\mathrm{fi}$ brous materials is demon- strated for the fi rst time. Within a few seconds, the non-destructive $\mathrm{C} 4 \mathrm{D}$ detector provides a pro $\mathrm{fi}$ le of the longetudinal physical homogeneity of the $\mathrm{fi}$ bre, as well as extra information regarding fi bre mophology and composition. In addition to the theoretical considerations related to the factors a ff ect the output signal, this work evaluates the properties of graphene fi bres using scanning $\mathrm{C} 4 \mathrm{D}$ following the manufac- turing process of wet-spinning. Furthermore, conductive graphene-coated $\mathrm{fi}$ brous materials and the e ff ectiveness of the coating and reduction procedures applied could be investigated. Apart from the application of C $4 \mathrm{D}$ in the monitoring of such processes, the feasibility of this small, highly sensitive and rapidly-responsive detector to monitor strain and elasticity responses of conductive and elastomeric composite fi bres for applications in motion sensing, biomedical monitoring, and stretchable electronics was also demonstrated.
\end{abstract}

\section{Keywords}

graphene, fibres, coated, capacitively, coupled, contactless, conductivity, detector, characterisation

\section{Disciplines}

Engineering | Physical Sciences and Mathematics

\section{Publication Details}

Cabot, J. M., Duffy, E., Currivan, S., Ruland, A., Jalili, R., Mozer, A. J., Innis, P. C., Wallace, G. G., Breadmore, M. \& Paull, B. (2016). Characterisation of graphene fibres and graphene coated fibres using capacitively coupled contactless conductivity detector. The Analyst, 141 (9), 2774-2782.

\section{Authors}

Joan M. Cabot, Emer Duffy, Sinead Currivan, Andres Ruland Palaia, Rouhollah Jalili, Attila J. Mozer, Peter C. Innis, Gordon G. Wallace, Michael C. Breadmore, and Brett Paull 


\section{Analyst}

\section{PAPER}

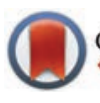

CrossMark \&cick for update-

Cite this: Analyst, 2016, 141, 2774

Received 10th December 2015 Accepted 17th February 2016

DOI: 10.1039/c5an02534f

www.rsc.org/analyst

\section{Characterisation of graphene fibres and graphene coated fibres using capacitively coupled contactless conductivity detector}

\author{
Joan M. Cabot, ${ }^{a, b}$ Emer Duffy, ${ }^{a}$ Sinéad Currivan, ${ }^{a}$ Andres Ruland, ${ }^{c}$ Rouhollah Jalili, ${ }^{c}$ \\ Attila J. Mozer, ${ }^{c}$ Peter C. Innis, ${ }^{c}$ Gordon G. Wallace, ${ }^{c}$ Michael Breadmore ${ }^{a, b}$ and \\ Brett Paull*a,b
}

The use of capacitively coupled contactless conductivity detection $\left(C^{4} D\right)$ for the characterisation of thin conductive graphene fibres, graphene composite fibres, and graphene coated fibrous materials is demonstrated for the first time. Within a few seconds, the non-destructive $C^{4} D$ detector provides a profile of the longetudinal physical homogeneity of the fibre, as well as extra information regarding fibre mophology and composition. In addition to the theoretical considerations related to the factors affect the output signal, this work evaluates the properties of graphene fibres using scanning $C^{4} D$ following the manufacturing process of wet-spinning. Furthermore, conductive graphene-coated fibrous materials and the effectiveness of the coating and reduction procedures applied could be investigated. Apart from the application of $C^{4} D$ in the monitoring of such processes, the feasibility of this small, highly sensitive and rapidly-responsive detector to monitor strain and elasticity responses of conductive and elastomeric composite fibres for applications in motion sensing, biomedical monitoring, and stretchable electronics was also demonstrated.

\section{Introduction}

Conducting fibres and textiles have become very attractive materials for the development of smart wearable garments and miniaturised electronic devices. ${ }^{1-5}$ With their flexible and durable structure, graphene-based fibres (GBFs) have been investigated for use within a wide variety of potential applications, such as conducting wires, fibre-based energy-storage devices, actuators, smart sensors, field emitters, microextraction phases, springs and as catalysts. ${ }^{6}$ The availability and liquid crystalline properties of the graphene oxide (GO) sheets, ${ }^{7,8}$ together with the use of wet-spinning technology ${ }^{9}$ has accelerated the progression of the manufacture of ultra-strong and electrically conducting GBFs. ${ }^{5,10-15}$ However, wet-spinning has some limitations in performance and requires precise control of formulation in the spinning dope dispersions, conditions

\footnotetext{
${ }^{a}$ Australian Centre for Research on Separation Science (ACROSS), School of Physical Sciences, Faculty of Science, Engineering and Technology, University of Tasmania, Private Bag 75, Hobart, TAS 7001, Australia. E-mail: brett.paull@utas.edu.au; Fax: +6136226 2858; Tel: +61 362266680

${ }^{b}$ ARC Centre of Excellence for Electromaterials Science (ACES), School of Physical Sciences, Faculty of Science, Engineering and Technology, University of Tasmania, Private Bag 75, Hobart, TAS 7001, Australia

${ }^{c}$ ARC Centre of Excellence for Electromaterials Science (ACES), AIIM Facility, Innovation Campus, University of Wollongong, Wollongong, NSW 2522, Australia
}

within the coagulation bath, with each step affecting final fibre morphology. Although the cost of processing GBFs is much lower than those of other carbon fibres, including carbon nanotube (CNT) containing fibres, the strength and electrical properties are poorer. These may be attributed to a number of factors, such as the crystallinity, large aspect ratio and packing density. Principally, lateral size to thickness ratio leads to increased folding of GO or reduced graphene oxide (rGO), generating numerous voids in the fibre and causing deterioration of the mechanical and electrical properties of the fibre. ${ }^{6,16-18}$ Therefore, considerable efforts have been made to improve these properties and extend the potential applications of GBFs.

Several spinning methods have been developed for GBFs, including dry-spinning, dry-jet wet-spinning, electrophoretic self-assembly, and film shrinkage or twisting. The resistance of GBFs changes with the diameter, and therefore, conductivity will be influenced by the cross-sectional area at every point. Thus, following fibre production, highly laborious inspection using microscopy or scanning electron microscopy (SEM) imaging becomes essential to determine morphological parameters to understand inherent electrical and mechanical properties. In order to develop the best spinning methodology for production of any particular GBF, rapid and effective online characterisation techniques, both during and post-production are required. 
In addition, alternative electrically conductive textiles can be made with a variety of fibres including cotton, polyester, and nylon, coated with electrically conductive elements, like carbon, aluminium, copper, gold, or silver. ${ }^{19-22}$ Deposition of gold or other metals on insulating fibres can produce conducting fibres, but also introduces an element of inflexibility, where stretching can easily damage a coating. This is a problem for applications in flexible or wearable devices. Carbon offers a promising alternative, particularly graphene, and has been applied in the development of novel conducting composites. $^{20,21}$ Following exfoliation from graphite, GO can be adsorbed onto a surface and converted to graphene by chemical reaction using hydrazine, hydroquinone, ascorbic acid, or sodium borohydride. ${ }^{23}$ However, adsorption and reduction of GO are critical steps that can drastically affect the final properties of a material or device composed of rGO. The conductivity of the final composite depends on the homogeneity and thickness of the coating along the surface. Coating exfoliation can cause a drastic decrease of conductivity or even detect no signal. Thus, and similarly to the GBFs mentioned above, on-line and non-destructive evaluation of the homogeneity of the conductive coating on composite fibres is essential for quality control during preparation.

Capacitively coupled contactless conductivity detection $\left(C^{4} \mathrm{D}\right)$ has been used extensively as a detector in separation science. ${ }^{24,25}$ In addition, the $\mathrm{C}^{4} \mathrm{D}$ cell has found alternate use as a characterisation tool of stationary phases for capillary columns, ${ }^{26}$ separation channels in microfluidic devices, ${ }^{27}$ flow measurements, ${ }^{28}$ and a recent electrical sensor for the simultaneous amplification and detection of specific sequence DNA. $^{29}$ The true benefit of this form of detection is related to its non-destructive character, providing characteristic information regarding the cell contents without disturbing the system under observation. $C^{4} \mathrm{D}$ has the ability to measure the resistance in series with the impedance of the coupling capacitance between two electrodes held within the detector, e.g. tubular electrodes placed around a non-conducting capillary (e.g. fused silica, PEEK or Teflon). After an AC voltage is applied to the actuator electrode, the current passes firstly through the capillary wall, the detection gap between the electrodes inside the capillary, and then to the pick-up electrode. Finally, the signal is further amplified and processed.

When used in scanning $\mathrm{C}^{4} \mathrm{D}$ mode $\left(\mathrm{sC}^{4} \mathrm{D}\right)$, a plot of detector response $(\mathrm{mV})$ versus position on column can provide a charge distribution profile along the capillary. ${ }^{26}$ This plot offers valuable information on the physico-chemical properties of the material within the capillary. In separation science, when comparing a multitude of capillary chromatography columns, $C^{4} \mathrm{D}$ provides a quality control step for capillary characteristics such as void space between particles, ${ }^{30}$ fabrication reproducibility, ${ }^{27}$ and optimisation of modification procedures. ${ }^{31,32}$ The majority of the $\mathrm{SC}^{4} \mathrm{D}$ applications have been applied to assess batch-tobatch reproducibility of analytical devices, with measurements limited to in-solution, i.e. a capillary or column wetted with a conducting liquid. However, $\mathrm{SC}^{4} \mathrm{D}$ has the potential to be highly useful in materials science. It can be employed in the development of composite fibres or coatings as a nondestructive characterisation step to provide valuable localised physico-chemical data, and it can be used at any stage in a material's development or application. This reduces the reliance on other more time consuming or expensive techniques such as electron microscopy, atomic force microscopy or spectroscopic methods. Herein, for the first time we explore the application of $\mathrm{C}^{4} \mathrm{D}$ as a characterisation tool for composite fibre manufacture control. Using a commercially available instrument, we demonstrate the potential of this high-throughput, non-invasive and non-destructive technique offers to the area of materials research, with a focus on carbon-based conducting fibres.

\section{Theory}

$\mathrm{C}^{4} \mathrm{D}$ can be described using a model which involves just one or two capacitors in series with a resistance, or a discrete element model wherein the two capacitors are divided into a multitude of resistor-capacitor elements within a network. In this instance the application of the $\mathrm{C}^{4} \mathrm{D}$ detector to characterise thin highly conductive fibres can be represented by Fig. 1. This allows measurement of the resistance of the fibre, $R_{\mathrm{F}}$, with consideration of the coupling capacitance of the wall and air gap, between the two electrodes, $\mathrm{E}_{1}$ and $\mathrm{E}_{2}$.

The $R_{\mathrm{F}}$ can be calculated by its length $(l)$, conductivity $(\kappa)$ and cross section area $(A)$ as shown in eqn (1).

$$
R_{\mathrm{F}}=\frac{l}{\kappa A}
$$

Additionally, coupling capacitance $\left(C_{\mathrm{cpl}}\right)$ can be defined by a combined series of capacitances, arising from the capillary wall and the air gap between electrodes. Since the system is based on a coaxial geometry, coupling capacitance of each capacitor, $C_{\mathrm{cpl}, \mathrm{i}}$, and can be calculated using the following eqn (2):

$$
C_{\mathrm{cpl}, \mathrm{i}}=\frac{2 \pi \varepsilon_{\mathrm{r}} \varepsilon_{0} w}{\ln \left(r_{2} / r_{1}\right)}
$$

where $\mathrm{w}$ is the electrode length, $\varepsilon_{0}$ and $\varepsilon_{\mathrm{r}}$ are the permittivity of a vacuum and the dielectric used, respectively, and $r_{1}$ and $r_{2}$ are the corresponding inner and the outer radii of the coaxial material. Subsequently, the coupling capacitance reactance $\left(X_{\text {cpl }}\right)$ will depend on the working frequency $(f)$, and this can be expressed as shown in eqn (3).

$$
X_{\mathrm{cpl}}=\frac{1}{2 \pi f C_{\mathrm{cpl}}}
$$

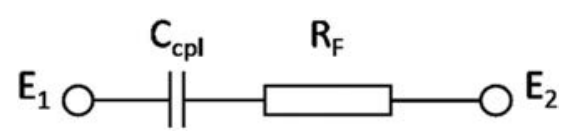

Fig. 1 Equivalent circuit diagram of a $C^{4} D$ cell without stray and leakage capacitance. $E_{1}$ and $E_{2}$ are the excitation and pick up electrode, respectively. $C_{\mathrm{cpl}}$ symbolises the total coupling capacitance due to the fused silica wall and air surrounding the fibre. $R_{\mathrm{F}}$ is the resistance of the fibre. 


\section{Experimental}

\section{Materials and instrumentation}

Poly(3,4-ethylenedioxythiophene):poly(styrenesulfonicacid) (PEDOT:PSS) pellets (Agfa Orgacon Dry, batch no. A06 0000BY), polycarbonate-based biocompatible thermoplastic polyurethane (AdvanSource Biomaterials Chronoflex C 80A) and dry expandable graphite flakes (3772, Asbury Graphite Mills, USA) were used as received. Bovine serum albumin (BSA), hydrazine monohydrate (64-65\%, reagent grade) and $N, N$-dimethylformamide (DMF) were purchased from SigmaAldrich (NSW, Australia), and tetrahydrofuran (THF), acetone and dimethylsulfoxide (DMSO) bought from Ajax Finechem (Thermo Fisher cientific, NSW, Australia). Water was purified using a Milli-Q plus system from Millipore (Bedford, MA, USA), to a resistivity of $18.2 \mathrm{M} \Omega \mathrm{cm}$. A Zetasizer Nano ZS particle analyser from ATA Scientific (Taren Point, NSW, Australia) fitted with a $632.8 \mathrm{~nm}$ red laser, and a $175^{\circ}$ backscatter detector was used to estimate the particle size distributions of aqueous GO suspensions.

A commercial capacitively coupled contactless conductivity detector, $C^{4} \mathrm{D}$ (TraceDec), was supplied by Innovative Sensor Technologies GmbH (Strasshof, Austria) with a capillary headstage that specifically accommodates $660 \mu \mathrm{m}$ fused silica capillary. Composite GBFs were introduced by vacuum into a fused-silica capillaries of $250 \mu \mathrm{m}$ I.D. (660 $\mu \mathrm{m}$ O.D.), and capillaries externally coated with rGO inserted into a 500 I.D. (660 $\mu \mathrm{m}$ O.D.) capillary to facilitate contactless longitudinal scanning. Capillaries were purchased from Polymicro Technologies (Phoenix, AR, USA). Prior to scanning, the capillaries were prepared by flushing with acetone, followed by deionised water, and then dried under compressed air. Detection parameters were selected according to the fibres' signal and capillary inner diameter as following: frequency, medium; voltage, $-12 \mathrm{~dB}$; gain, 50\%; off set, 0 (except where stated). Data was collected and processed with TraceDec Monitor Software v0.08c using analog output with an AD converter. For GBFs scanning a syringe pump from Harvard Apparatus (Holliston, MA, USA) was used to push the fibre through the $C^{4} \mathrm{D}$. Settings of the syringe pump were selected in order to obtain a constant speed of $0.50 \mathrm{~cm} \mathrm{~min}^{-1}$ corresponding a 240 measurements per mm. Images of GBFs were taken with a USB microscope (AD7013MZT Dino-Lite Premier and AM4515T5 Dino-Lite Edge). Fig. 2 shows the schematic set-up for scanning GBFs. Scans were done at room temperature. For comparative results, measurements were done at same day in order to mitigate the temperature effect in the results. Stress and release of graphene fibres were applied moving the platform until the desired length for the specified period of time.

Scanning electron microscopy (SEM) imaging was carried out on sections of fibre samples using a Hitachi SU70 instrument (Hitachi High Technologies America, USA) operating at $1.5 \mathrm{kV}$. Samples were sputter coated with a thin (ca. $4 \mathrm{~nm})$ layer of Pt prior to imaging using a Baltec Sputter Coater (SCD 050, Leica Microsystems, Liechtenstein).

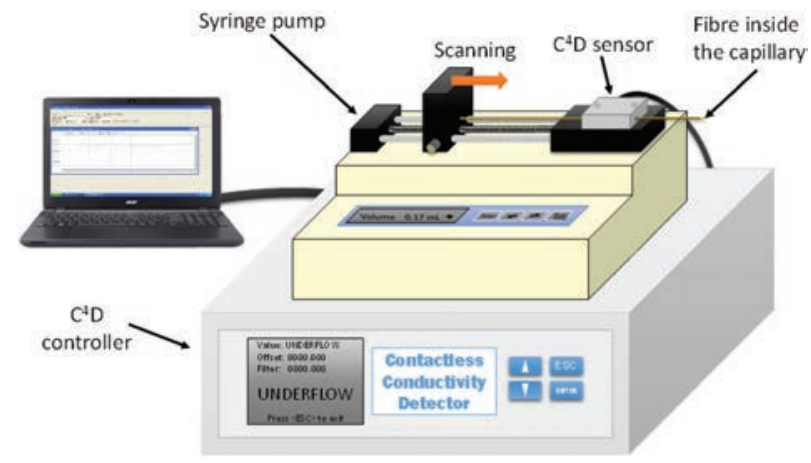

Fig. 2 Schematic representation of the scanning setup. The graphenebased fibre was introduced by vacuum into a fused-silica capillary (not shown). Acquisition of data was performed at $20 \mathrm{~Hz}$, with the syringe pump velocity at $0.50 \mathrm{~cm} \mathrm{m^{-1 }}$ (equivalent to 240 measures per $\mathrm{mm}$ ). Detection parameters were selected depending on the intensity shown on the $C^{4} D$ controller.

\section{Graphene Fibre fabrication and wet-spinning}

Aqueous dispersions of PEDOT:PSS pellets of concentration $25 \mathrm{mg} \mathrm{mL}{ }^{-1}$ were prepared via homogenisation, followed by bath sonication using a previously reported method. ${ }^{11}$ Dry expandable graphite flakes were treated and used as a precursor for graphene oxide (GO) synthesis following previously described methods. ${ }^{9,13}$

PEDOT:PSS and rGO fibres were fabricated at room temperature using wet-spinning apparatus..$^{9,11,12}$ For comparative purposes, wet-spinning was also carried out extruding at $10.0 \mathrm{~mL} \mathrm{~h}^{-1}$ through a needle (19 gauges) into a coagulation bath with $\mathrm{CaCl}_{2}$, water/ethanol and reduction agent, and stored overnight at $80{ }^{\circ} \mathrm{C}$. Subsequently, fibres were washed with water, and air dried under tension (0.025 N). Post-treatment was carried out using DMF. The contact conductivity values were calculated following eqn (1). Resistances of the fibres were measured by 4 point probe using a gap of $0.23 \mathrm{~cm}$.

In the case of $13 \mathrm{wt} \%$ polyurethane (PU) and PEDOT:PSS (PU/PEDOT:PSS) composite fibres, a wet-spinning procedure was performed as described previously. ${ }^{14}$

\section{GO coating}

Graphene oxide was prepared using a $2 \mathrm{mg} \mathrm{mL}^{-1}$ suspension (batch no. 247SG) as described above, with an average sheet size of $250 \mathrm{~nm}$. The preparation of rGO-coated materials was carried out using fused silica capillaries (Polymicro Technologies, Phoenix, AZ, USA, with a $150 \mu \mathrm{m}$ I.D. and a $365 \mu \mathrm{m}$ O.D.), and donated lengths of red human hair. The capillaries were rinsed with water, acetone and air-dried, and placed for $30 \mathrm{~min}$ in a sample tube filled with the $2 \mathrm{mg} \mathrm{mL}^{-1}$ suspension of GO. Following removal of the capillary from the suspension, it was dried at $50{ }^{\circ} \mathrm{C}$ for $15 \mathrm{~min}$. Capillaries were then hung over hydrazine overnight to facilitate reduction of GO via vapours. Subsequently, they were washed $(3 \times)$ with deionised water and air-dried prior to characterisation.

The strands of hair was first washed with soapy water by rubbing between the fingers for $1 \mathrm{~min}$, followed by $3 \times$ washing 
with deionised water. A strand of hair was then submerged in a $0.5 \mathrm{wt} \% \mathrm{BSA}$ solution for $10 \mathrm{~min}$ at room temperature followed by drying in the oven at $30^{\circ} \mathrm{C}$ for $10 \mathrm{~min}$. BSA was dissolved in deionised water and utilised immediately. Hair was then rinsed $3 \times$ with water to remove any residual BSA molecules. Multiple layers of coatings for hair samples were prepared by repeating the steps above. This was achieved by submerging the BSA-coated hair in the GO suspension for $30 \mathrm{~min}$, followed by drying in the oven at $50{ }^{\circ} \mathrm{C}$ for $15 \mathrm{~min}$. The hair was then hung over hydrazine to facilitate reduction, washed with deionised water $(\times 3)$, and the process was repeated on controlled sections of the hair to build up a gradient coating of rGO along the same length of hair. The same experimental procedure was carried out without the use of BSA for comparative purposes.

Blank capillary and hair samples were also prepared in a similar manner, where they were washed and exposed to hydrazine vapours according to the procedures outlined above. Fused silica capillary coating was achieved both with and without BSA. Therefore, BSA was not used for coated capillaries as it simplified the procedure.

\section{Results and discussion}

\section{$C^{4} D$ output signal in high conductive materials}

Since its discovery, the theoretical basis and circuit scheme approximations of a $\mathrm{C}^{4} \mathrm{D}$ detector have been described in depth in numerous works. ${ }^{28,29,33-46}$ However, the nature of the $\mathrm{C}^{4} \mathrm{D}$ output signal for the application developed in this work, is worthy of further consideration, as it differs from that described in previous works published. This section explains the mechanism using empiric calculations, as to how the $C^{4} \mathrm{D}$ works in the measurement of thin, and highly conductive GBFs and coatings.

Typically, in all $\mathrm{C}^{4} \mathrm{D}$ approaches the capillary is full of a liquid with very low conductivity, for instance $0.2-1.2 \mathrm{mS} \mathrm{cm}^{-1}$ for a conventional capillary electrophoresis technique. Consequently, the impedance attributed to the coupling capacitance is derived from the capillary wall, which is low and generally constant. The conductivity of the solution is then the variable element, where variation in ionic concentration in bulk solution increases or reduces the resistivity, which is consequently observed within the voltage signal. Herein, the buffer is supplanted for a thin and highly conductive GBF, leaving an air gap between fibre and capillary wall. This change in configuration produces two main alterations in the system. The first, the resistance between the electrodes decreases around 1000fold compared to a $100 \mu \mathrm{m}$ I.D. capillary containing a buffer solution. The second, the coupling capacitance reactance increases due to the fibre containing capillary containing a considerable volume of air.

GBFs are approximately $10-400 \mathrm{~S} \mathrm{~cm}^{-1}$ with diameters between 50-200 $\mu \mathrm{m} .{ }^{6}$ Following eqn (1) and supposing an electrode gap of $10 \mathrm{~mm}, R_{\mathrm{F}}$ can range from $c a$. 0.01 to $5 \mathrm{k} \Omega$. The variation in thickness between fibres and indeed along the fibre, causes variations in resistance. For instance, the larger the cross-sectional area of the conductor, the more electrons per unit length are available to carry the current. As a result resistance is lower, conductivity higher, and hence the $C^{4} D$ signal higher. Using a capillary of 250 inner and $660 \mu \mathrm{m}$ outer diameter, and a $3 \mathrm{~mm}$ long electrode, capacitance can be estimated using eqn (2). For the capillary wall, $C_{\text {cpl,wall, }}$ is estimated to be $0.55 \mathrm{pF}$. This capacitance will be relatively constant as wall thickness is constant along the capillary. In the case of the air capacitance, $C_{\mathrm{cpl} \text {,air }}$, this can vary depend on the diameter of the fibre. Supposing that the fibre varies from 50 to $200 \mu \mathrm{m}$, the capacitance ranges from 0.1 to $0.75 \mathrm{pF}$, and thus results in an impedance $\left(X_{\mathrm{cpl}, \text { air }}\right)$ of between $400-3000 \mathrm{k} \Omega$ using $500 \mathrm{kHz}$ (eqn (3)). However, the lower the frequency, the higher the impedance and vice-versa.

Using this information, and assuming a constant composition along the length of a fibre, changes in $\mathrm{C}^{4} \mathrm{D}$ signal are essentially due to variations in fibre diameter causing variations in $R_{\mathrm{F}}$ and $X_{\mathrm{cpl}}$. Therefore, it is possible to characterise the GBF and graphene coated fibres by monitoring the signal received in the pick-up electrode while the sample is being scanned.

\section{Characterisation of fibre production}

In order to compare responses in $\mathrm{C}^{4} \mathrm{D}$ signal behaviour, two different GBFs were produced. The first fibre was made using $100 \%$ of rGO, and the second using $50 \%$ of PEDOT:PSS and $50 \%$ rGO. Fabrication and wet-spinning were carried out as described within the Experimental section. However, the second fibre was air-dried and reduced without tension, producing an irregular and rough structure. Fig. 3 shows the output voltage of the two different conductive fibres along their length. Each GBFs was scanned 5 times length to length, under the same settings. These 5 scans for each fibre are overlaid within Fig. 3. Fig. 3(a) shows the response traces for the rGO fibre, and Fig. 3(b) for those achieved scanning the PEDOT:PSS + rGO composite fibre.

Looking at the profile of the signals, the rGO fibre is smooth and with zones of relatively uniform signal, whereas the composite fibre shows a much more irregular response. Note, that to the naked eye in the photo insert of Fig. 3(b), that the composite fibre shape is considerable coarser than the rGO fibre, a property that was clearly captured within the $\mathrm{SC}^{4} \mathrm{D}$ response traces. As can be seen, $\mathrm{sC}^{4} \mathrm{D}$ precision was excellent over the five separate scans, with a resulting \%RSD of 1.7 and 0.6 for rGO and the composite fibre, respectively. However, the signal scale for rGO fibre is 5 times bigger, from 1000 to $2000 \mathrm{mV}$. The average voltage for the entire length of the rGO fibre showed more than 7 times greater variation than the composite fibre, reporting $1341 \pm 202 \mathrm{mV}$ and $866 \pm 27 \mathrm{mV}$, respectively. Therefore, the composite fibre in Fig. 3(b) was more homogeneous, despite its irregular appearance. Correspondingly, tension during the air-drying process of the rGO fibre might have caused elongation, and a reduction in diameter of some parts of the fibre. On the contrary, no strain during the air-drying process produced a rough and irregular 


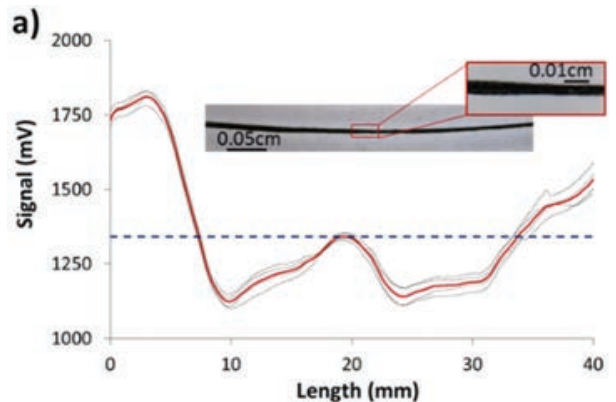

b)

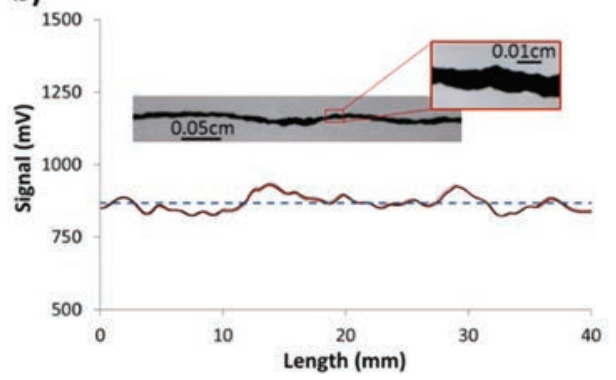

Fig. 3 Signal of 5 consecutive scanning with respect to the fibre length of (a) rGO and (b) PEDOT:PSS + rGO (1:1). Solid red line shows the average signal of all 5 scans. Dashed blue line shows signal average of the entire fibre. $C^{4} D$ programmed parameters: frequency, medium; voltage, $-6 \mathrm{~dB}$; gain, $50 \%$ and offset, 0 . Images of the fibres were taken using a Dino-Line microscope.

fibre, which may affect the physical properties. Ensuring a homogenous dispersion of the composite mixture during the wet-spinning process, conductivity should be high and constant along the fibre. Thus, the most likely reason for these variations within the in $\mathrm{C}^{4} \mathrm{D}$ scans is changes in the fibre thickness. If the fibre is thicker, coupling capacitance of the air gap will increase, causing a decrease in impedance and therefore an increase of the output signal.

Looking at the appearance of both fibres using a Dino-Lite microscope, cylindrical shapes were revealed for the entire section. In order to have a longitudinal thickness profile for both fibres, they were cut into sample lengths of approximately $1 \mathrm{~cm}$ and imaged using FE-SEM. Fig. 4(a) and (b) show two consecutive micrographs separated by $2 \mathrm{~mm}$ for each fibre. These images illustrate the GBF structure, as well as local thickness variability. Unlike rGO fibres, the SEM micrographs of the composite fibres confirmed large variabilities in diameter along the fibre length, ranging from 42.6 and $128.0 \mu \mathrm{m}$. Subsequently, thickness profiles could only be demonstrated for rGO fibre (Fig. 4(c). Note the close similarity in the longitudinal thickness profile measured manually using FE-SEM and the signal obtained using $\mathrm{SC}^{4} \mathrm{D}$ (Fig. 3(a)).

\section{Formulation of graphene composite fibres}

To investigate the effect of fibre composition on $\mathrm{C}^{4} \mathrm{D}$ response more fully, $\mathrm{sC}^{4} \mathrm{D}$ signal was used to measure four GFBs with formulations of $0,25,50,75 \mathrm{wt} \%$ PEDOT:PSS in rGO, labelled as Fibre $1,2,3$ and 4 , respectively. Theoretically, different a)
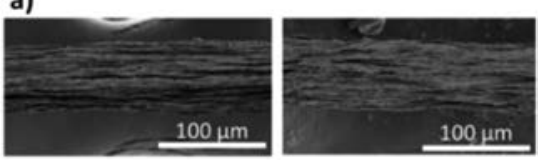

b)

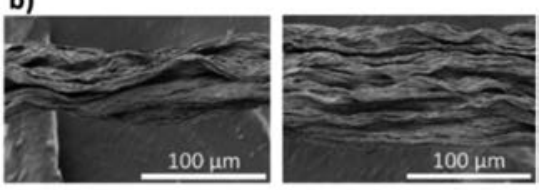

c)

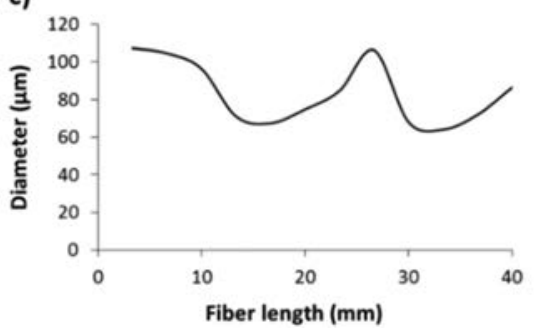

Fig. 4 SEM images of the (a) PEDOT:PSS + rGO (1:1), and (b) rGO fibres. Consecutive images were taken within $2 \mathrm{~mm}$ of distance. (c) Diameter profile of rGO fibre with respect to the length.

material formulations in conductive composites provide changes in the morphology and the conductive properties of the final product. According to the $\mathrm{C}^{4} \mathrm{D}$ theory discussed above, the observed response should primarily reflect changes in the fibre thickness for the composite fibres, rather than conductivity differences.

Herein the $\mathrm{SC}^{4} \mathrm{D}$ profiles showed a good level of longitudinal homogeneity along the fibre, with no more than $20 \mathrm{mV}$ of variation for a single scan within $20 \mathrm{~mm}$ of fibres. The average signals obtained were $952 \pm 17,620 \pm 14,648 \pm 7,684 \pm 18 \mathrm{mV}$ for Fibres 1, 2, 3 and 4, respectively. Assuming that dispersions of the composite mixture prior to wet-spinning were homogeneous, the results indicate relatively constant thicknesses. Comparing the four GBFs, responses appeared relatively constant, except for Fibre 1, which was higher than the other fibres. This increased output signal suggested that this fibre was thicker than the others.

In order to confirm this, the base area of all fibres was determined. Inspecting the shape of the fibre using a DinoLite microscope, it was found that GBFs were not completely spherical and had similar structure to an irregular twisted rectangle. Thus, the thickness of four samples of each fibre were measured using SEM. Three measurements of approximately 25 micrographs of each fibre were made. The cross sectional area of each fibre was then calculated according to its geometry (i.e. rectangular). The obtained base-area values were 8087, 6294, 7968 and $10848 \mu^{2}$ for Fibres 1, 2, 3 and 4, respectively. However, surprisingly, in this case, despite the strong influence that impedance has in the $\mathrm{C}^{4} \mathrm{D}$ measurements, responses did not directly correlate with fibre diameter. Therefore, as these results could not be fully attributed to the changes in coupled capacitance, the attributable reason might 

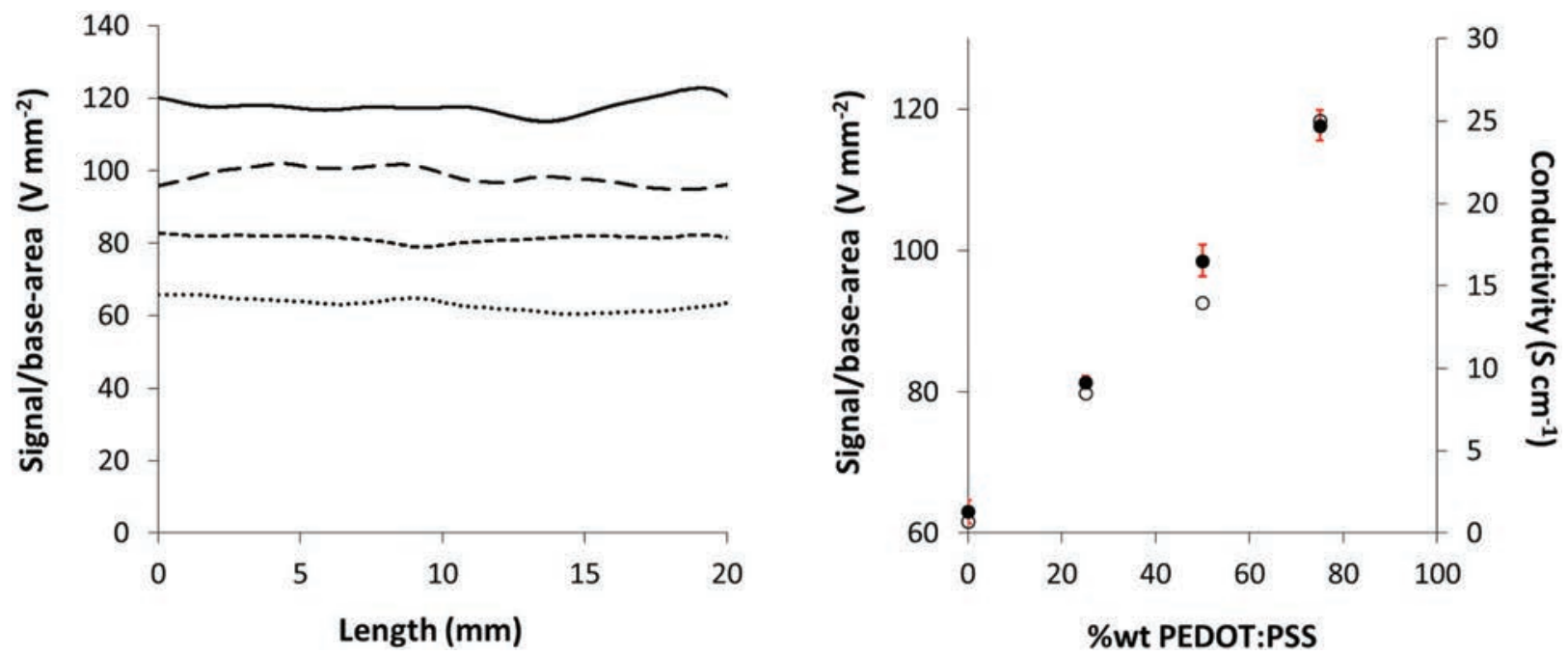

Fig. 5 Intrisic $C^{4} D$ signal obtained for PEDOT:PSS + rGO composite fibres. Fibre 1: 0\% PEDOT:PSS; Fibre 2: 25\% PEDOT:PSS; Fibre 3: 50\% PEDOT: PSS; Fibre 4: 75\% PEDOT:PSS in rGO; (a) signal by thickness area versus length of the GBFs. Legend: Fibre 1, . . . . ; Fibre 2, - - -; Fibre 3, - -; Fibre $4,-$. (b) Average signal/base-area as a fuction of the wt\% of PEDOT:PSS in rGO (filled dots) and comparison with contact conducitivity values (empty dots).

be increments in conductivity, permittivity of the fibres, or both together.

To obtain information to support this notion, the signal was normalised by base area. Fig. 5(a) shows how the signal/ area decreases when reducing the amount of PEDOT:PSS in the composite fibre. Shown in Fig. 5(b) is the correlation between the average signal/area versus wt $\%$ of PEDOT:PSS. Since PEDOT:PSS is more conductive than rGO, the PEDOT: PSS loading increases the conductivity of the fibre, which is shown in the normalised $\mathrm{SC}^{4} \mathrm{D}$ response. Likewise, signal by area detected, clearly demonstrated a linear increase. A comparison with contact conductivity values (empty dots in Fig. 5(b)) shows a scalable relationship with contactless detection. Therefore, the very clear and linear correlation observed demonstrates the technique's ability to measure changes in fibre composition (conductivity).

\section{Evaluation of GO coatings}

As a demonstration of the capability offered by $\mathrm{C}^{4} \mathrm{D}$, two further rGO-modified (coated) materials were prepared. Firstly, human hair was selected as a strong and flexible fibrous structure for rGO coating, due to ease of treatment to impart a desired functionality or property to the surface. ${ }^{47}$ BSA, which serves as a universal adhesive to facilitate the adsorption of GO nanosheets, ${ }^{21,48}$ can be immobilised via hydrophobic adsorption to introduce positive charges on the hair surface. Subsequently, the BSA-functionalised hair allows the selfassembly of negatively charged GO sheets. Secondly, $360 \mu \mathrm{m}$ O.D. fused polyimide coated silica capillary was selected for its strength and thickness homogeneity, and it can be easily placed within a $500 \mu \mathrm{m}$ I.D. capillary for $\mathrm{C}^{4} \mathrm{D}$ scanning purposes. The procedure of coating and chemical reduction of GO on the hair and capillary samples in this work could readily be observed, as in both instances the rGO turned the colour black, suggesting that the rGO was successfully adsorbed on the surface. The surface of either hair or capillary did not show exfoliation of rGO during routine handling or bending.

rGO coating on hair. The $\mathrm{SC}^{4} \mathrm{D}$ profile of the entire length of a blank hair (no rGO coating) showed underflow response for all programmed settings. This demonstrates that resistance ( $R_{\mathrm{F}}$ from circuit in Fig. 1) between electrodes is too high to be able to pick up signal from the actuator electrode. $\mathrm{sC}^{4} \mathrm{D}$ of hair + rGO (but without BSA) showed the same behaviour, since GO cannot be adsorbed on the surface of the hair prior to its reduction. However, a minimal response was detected for the blank hair with BSA, confirming that BSA was adsorbed on the surface. When hair + BSA + rGO was produced it was evident that a considerable level of coating was achieved, with a corresponding change in colour from red to black. SEM micrographs in Fig. 6(a) and (b) show the surface of the hair, before and after one coating cycle, respectively. $\mathrm{sC}^{4} \mathrm{D}$ signals are shown in Fig. 6(c). The traces shown indicate that the coating was achieved and that each coating cycle increased the $C^{4} \mathrm{D}$ response accordingly. The $\mathrm{rGO}_{x}$ in this figure also shows each subsequent coating, where ' $x$ ' is the number of rGO cycles performed. Since increasing conductivity does not dominate the signal, the traces shown are a reflection of the fact that each subsequent coating caused increments in hair thickness as well as conductivity. Interestingly, the recorded traces showed broad peaks rather than flat profiles, indicating a non-uniform coating was achieved, something which was not easily detectable by visual inspection or SEM.

rGO coating on fused silica capillary. For a blank capillary the $\mathrm{SC}^{4} \mathrm{D}$ profile showed underflow output across the entire length of capillary. Fig. 7(a) shows the exterior of the blank capillary. When the capillary was coated with rGO, the colour turned to black suggesting efficient coating. SEM imaging revealed poor GO adsorption on the inner walls of the capillary 

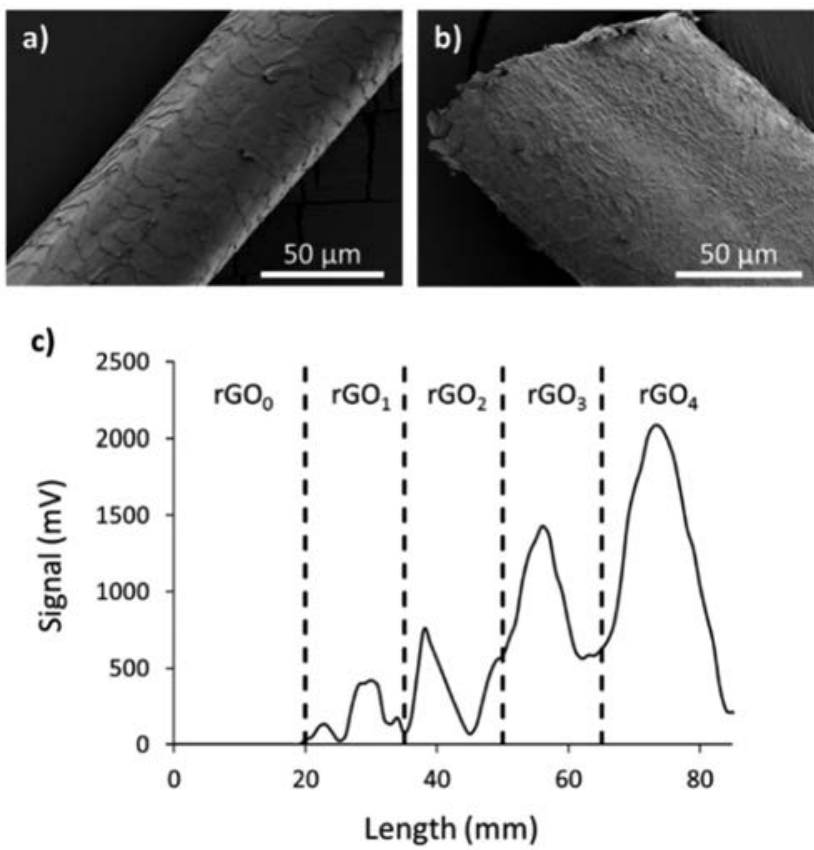

Fig. 6 Characterisation of rGO coating on human hair. Above, 2 SEM images showing modified hair samples: (a) blank hair; (b) hair with one coating cycle $\left(\mathrm{rGO}_{1}\right)$. Below: (c) $\mathrm{C}^{4} \mathrm{D}$ signal profile with respect to the length. Subscripts of $\mathrm{rGO}_{x}$ show the coating cylces. Operating $\mathrm{C}^{4} \mathrm{D}$ conditions: frequency, $2 \times \mathrm{HIGH}$; voltage, $-6 \mathrm{~dB}$; gain, $50 \%$ and offset, 0 .

(Fig. 7(b)), which is obviously due to the negative charge from the silanol groups on the inner capillary walls. However, the SEM images revealed significant rGO coating on the polyimide (exterior) surface of the capillary via $\pi-\pi$ and weak electrostatic interactions (Fig. 7(c) and (d)). Following this surface modification, the $\mathrm{SC}^{4} \mathrm{D}$ signal revealed a relatively constant and smooth surface modification had been achieved between 15 and $80 \mathrm{~mm}$, as shown in Fig. 7(e).

\section{Output signal under tensile strain for elastomeric fibres}

In 2014, Seyedin et al. ${ }^{14}$ achieved the production of conducting elastomeric composite fibres, with strain sensing capability, combining elastomeric polyurethane (PU) and high conductive PEDOT:PSS. They demonstrated the response of cyclic stretching, as well as change in resistance once strain was applied. Despite the stretching abilities, they observed that strain in PU/PEDOT:PSS fibres provoked reversible and irreversible networks which might contribute to gain or loss of conductivity. This fibre showed a resistance of $5.4 \mathrm{k} \Omega$ before stretching, $40 \mathrm{k} \Omega$ under $50 \%$ strain and $500-600 \mathrm{k} \Omega$ under $100 \%$. Thus, this particular elastomeric composite offered great potential for applications in motion sensing, biomedical monitoring, and stretchable electronics. However, there was a need to detect and monitor the strain actuation 'on-fibre'. Thus herein the $C^{4} \mathrm{D}$ approach was further investigated as a tool for 'on-fibre' strain sensing applications.

For this study, PU/PEDOT:PSS fibres with $13.0 \mathrm{wt} \%$ of PEDOT:PSS were prepared as previously reported, ${ }^{14}$ and $\mathrm{C}^{4} \mathrm{D}$
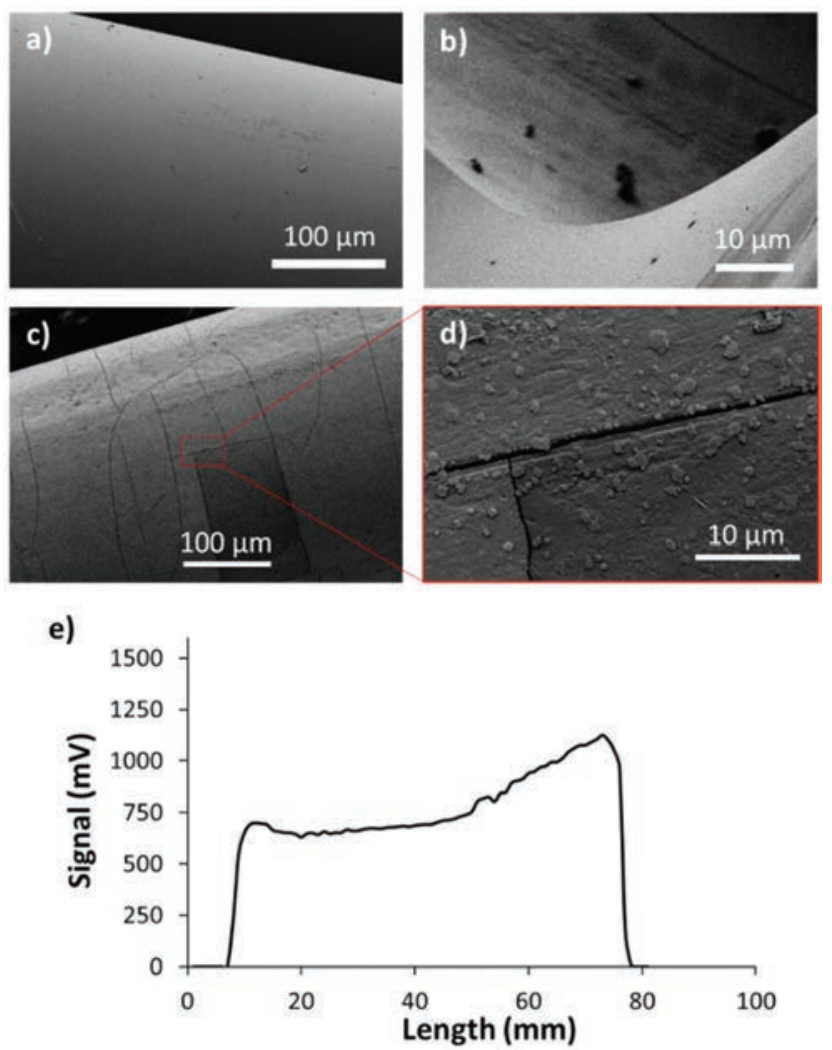

Fig. 7 Characterisation of rGO coating on fused silica capillary. Above, 4 SEM images showing the interior and exterior of the capillary: (a) exterior blank; (b) inside of the capillary after the coating, (c) and (d) outside after the coating. Below: (e) $C^{4} D$ signal profile with respect to the length. Operating $C^{4} \mathrm{D}$ conditions: frequency, $2 \times \mathrm{HIGH}$; voltage, $-18 \mathrm{~dB}$; gain, $50 \%$ and offset, 0 .

was used to monitor physical stress. Fig. 8 represents the variation of the $C^{4} D$ signal with respect to the (a) strain, and (b, c) time. Shown in Fig. 8(a) is a profile of the change in voltage with respect to the \% applied strain, where each stretching zone is denoted by a number between brackets in this figure. Initially, the composite was relaxed and a maximum voltage was shown.

Once the fibre was under tension, stretching was initiated and consequently the signal decreased (Fig. 8(a), zone 1). Variations in the signals were attributed to changes in diameter, brought on by the applied stretching. However, strain produces reversible and irreversible network breakages, which can contribute to a gain in resistance, causing a loss of conductivity and a hence decrease of $\mathrm{C}^{4} \mathrm{D}$ signal. Since resistance decreases up to hundreds of $\mathrm{k} \Omega, C_{\mathrm{cpl}}$ is then comparable with resistance. When stretching reached approximately $110 \%$ of length, the voltage remained constant (Fig. 8(a), zone 2). This might be due to all reversible and irreversible networks being broken, with stretching only affecting the non-conductive polyurethane matrix. Finally, in zone 3 of Fig. 8(a), strain exceeded its limits and the composite fibre snapped causing a drastic drop of signal.

Fig. 8(b) shows the relationship of signal versus time, while a $6 \mathrm{~cm}$ fibre was under $100 \%$ tensile strain for $1 \mathrm{~s}$ and then 

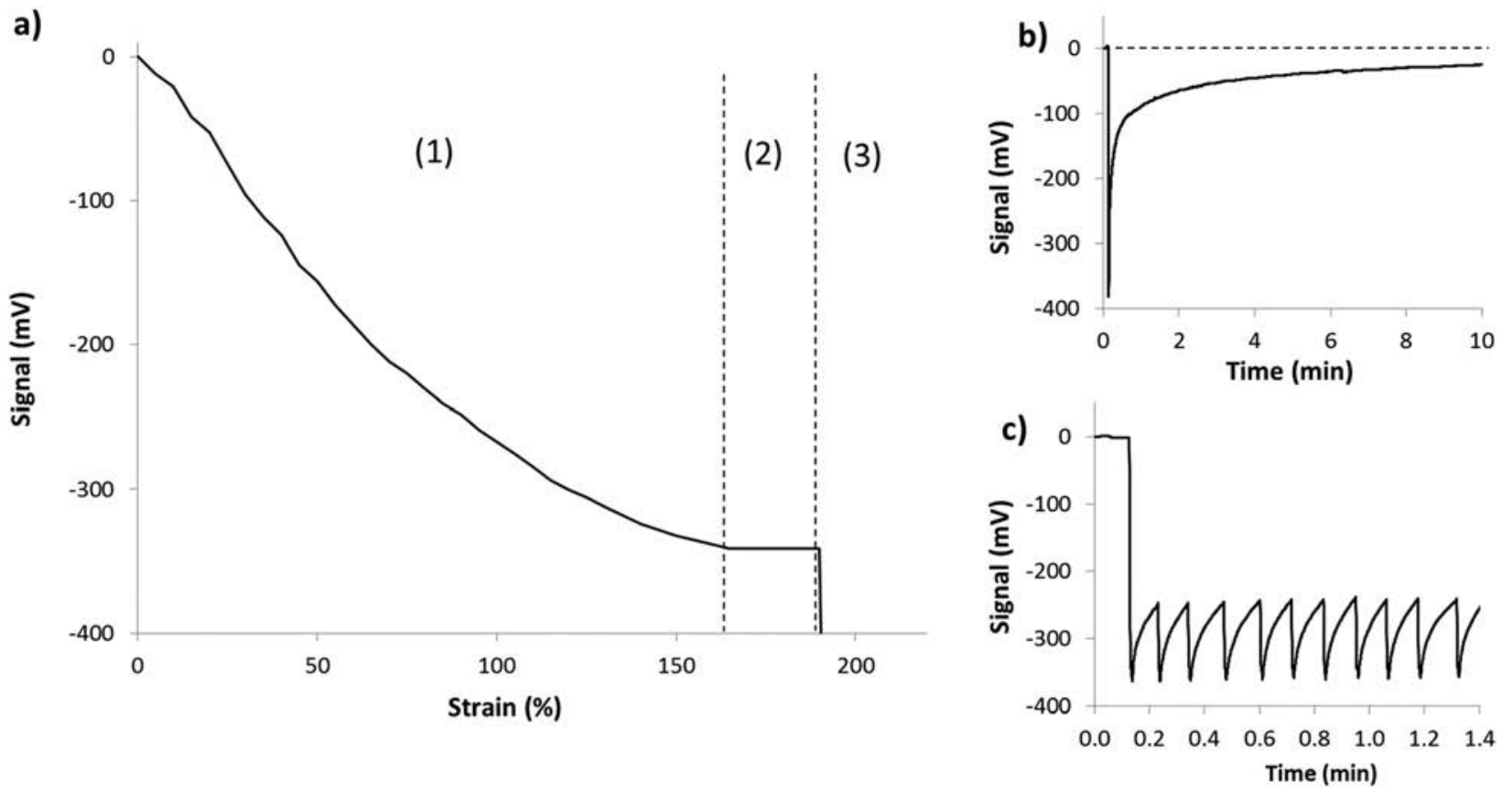

Fig. 8 Signal change by strain for PU/PEDOT:PSS composite fibres with 13.0 wt\% PEDOT:PSS. (a) Signal under a continuous stretching. Fibre length, $3.0 \mathrm{~cm}$. Numbers illustrate stretching zones. (b) Signal monitoring of $100 \%$ strain for $1 \mathrm{~s}$ and relaxation. (c) Signal monitoring of 10 stretching events, every 6-7 s, each stretching consists in $100 \%$ strain for $1 \mathrm{~s}$ and tension release. $C^{4} D$ programmed parameters: frequency, medium; voltage, $-18 \mathrm{~dB}$ (a) and $-12 \mathrm{~dB}(\mathrm{~b}, \mathrm{c})$; gain, $50 \%$ and offset, 0 .

released. It can be clearly seen that signal firstly decreased drastically. Secondly, when the composite fibre was released, the signal increased as thickness increased, and reversible networks come in contact again stabilising conduction paths within the fibre. Since the fibre is not completely elastic, after 10 minutes the signal had not returned to the initial level. Clearly, irreversible network breakages caused reduction of the conduction paths after certain repetitive strain applications. This method provides a new means to test such materials for durability and electrochemical properties under strain. Recoveries obtained for this PU/PEDOT:PSS are 50\% in $4.5 \mathrm{~s} ; 75 \%$ in $39.8 \mathrm{~s}$ and $93.2 \%$ in 10 minutes.

Voltage signal versus time during $100 \%$ strain and tension release over 10 events, every 6-7 s, was monitored and the profile is shown in Fig. 8(c). Initially, the composite fibre was relaxed and the voltage remained stable. When the fibre was stretched, the voltage decreased rapidly, and once the tension was released the voltage increased again. The average minimum voltage recorded was $-361 \pm 2 \mathrm{mV}$, with immediate recovery $(25 \%)$ at $3.7 \pm 0.2 \mathrm{~s}$. The high sensitivity and precision, fast response, and high rate of signal/noise demonstrate that the $C^{4} \mathrm{D}$ detector is suitable as a rapid response detector in stretchable material applications.

\section{Conclusions}

Capacitively coupled contactless conductivity detection has been demonstrated as a new tool to provide simple, low cost and rapid information on the structure and chemical composition of conducting fibres, specifically here graphene, composite and graphene coated fibres, and may be used as an inexpensive screening, and potentially on-line tool during material development, allowing improvements in synthesis strategies and resulting properties. This rapid, sensitive and efficient commercial instrument has been proven to distinguish different structures based on thickness variations and changes in morphology of a series of composite materials. Moreover, $\mathrm{C}^{4} \mathrm{D}$ has been able to quickly scan and detect the reduced graphene oxide coating homogeneity on two different materials.

The application of $\mathrm{C}^{4} \mathrm{D}$ in the strain-responses of $\mathrm{PU} /$ PEDOT:PSS elastomeric composite fibres, has also been demonstrated. Fast and precise responses were achieved in strain sensing of fibres, proving that $C^{4} D$ may be a future option as a micro detector integrated for bionics, such as motion sensors and biomedical monitoring.

\section{Acknowledgements}

This work is funded by the Australian Research Council (ARC) Centre of Excellence program and the Universities of Tasmania (UTas) and Wollongong (UOW). Prof Gordon Wallace is grateful to the ARC for support under the Australian Laureate Fellowship scheme (FL110100196). Funding from the Australian Research Council Centre of Excellence Scheme (Project Number CE 140100012) is gratefully acknowledged. 


\section{Notes and references}

1 A. I. S. Neves, T. H. Bointon, L. V. Melo, S. Russo, I. de Schrijver, M. F. Craciun and H. Alves, Sci. Rep., 2015, 5, 9866.

2 Y. Qin, X. Wang and Z. L. Wang, Nature, 2008, 451, 809813.

3 S. Chen, W. Ma, Y. Cheng, Z. Weng, B. Sun, L. Wang, W. Chen, F. Li, M. Zhu and H.-M. Cheng, Nano Energy, 2015, 15, 642-653.

4 S. Seyedin, J. Razal, P. C. Innis, A. Jeiranikhameneh, S. Beirne and G. G. Wallace, ACS Appl. Mater. Interfaces, 2015, 7, 21150-21158.

5 S. H. Aboutalebi, R. Jalili, D. Esrafilzadeh, M. Salari, Z. Gholamvand, S. Aminorroaya Yamini, K. Konstantinov, R. L. Shepherd, J. Chen, S. E. Moulton, P. C. Innis, A. I. Minett, J. M. Razal and G. G. Wallace, ACS Nano, 2014, 8, 2456-2466.

6 F. Meng, W. Lu, Q. Li, J.-H. Byun, Y. Oh and T.-W. Chou, Adv. Mater., 2015, 27, 5113-5131.

7 D. R. Dreyer, S. Park, C. W. Bielawski and R. S. Ruoff, Chem. Soc. Rev., 2010, 39, 228-240.

8 J. E. Kim, T. H. Han, S. H. Lee, J. Y. Kim, C. W. Ahn, J. M. Yun and S. O. Kim, Angew. Chem., Int. Ed., 2011, 50, 3043-3047.

9 R. Jalili, S. H. Aboutalebi, D. Esrafilzadeh, R. L. Shepherd, J. Chen, S. Aminorroaya-Yamini, K. Konstantinov, A. I. Minett, J. M. Razal and G. G. Wallace, Adv. Funct. Mater., 2013, 23, 5345-5354.

10 A. J. Granero, P. Wagner, K. Wagner, J. M. Razal, G. G. Wallace and M. In Het Panhuis, Adv. Funct. Mater., 2011, 21, 955-962.

11 R. Jalili, J. M. Razal, P. C. Innis and G. G. Wallace, Adv. Funct. Mater., 2011, 21, 3363-3370.

12 R. Jalili, J. M. Razal and G. G. Wallace, J. Mater. Chem., 2012, 25174-25182.

13 D. Li, M. B. Müller, S. Gilje, R. B. Kaner and G. G. Wallace, Nat. Nanotechnol., 2008, 3, 101-105.

14 M. Z. Seyedin, J. M. Razal, P. C. Innis and G. G. Wallace, Adv. Funct. Mater., 2014, 24, 2957-2966.

15 Z. Xu, H. Sun, X. Zhao and C. Gao, Adv. Mater., 2013, 25, 188-193.

16 S. Pei and H. M. Cheng, Carbon N. Y., 2012, 50, 32103228.

17 H. Cheng, C. Hu, Y. Zhao and L. Qu, NPG Asia Mater., 2014, 6, e113.

18 Z. Xu and C. Gao, Acc. Chem. Res., 2014, 47, 1267-1276.

19 H. M. Lee, S.-Y. Choi, A. Jung and S. H. Ko, Angew. Chem., Int. Ed., 2013, 52, 7718-7723.

20 B. Liang, L. Fang, Y. Hu, G. Yang, Q. Zhu and X. Ye, Nanoscale, 2014, 6, 4264-4274.

21 Z. Lu, C. Mao and H. Zhang, J. Mater. Chem. C, 2015, 3, 4265-4268.
22 G.-W. Huang, H.-M. Xiao and S.-Y. Fu, Nanoscale, 2014, 6, 8495.

23 Y. Zhu, S. Murali, W. Cai, X. Li, J. W. Suk, J. R. Potts and R. S. Ruoff, Adv. Mater., 2010, 22, 3906-3924.

24 P. Kubáň and P. C. Hauser, Electrophoresis, 2009, 30, 176188.

25 J. A. F. da Silva, C. L. do Lago, D. P. de Jesus and W. K. Tomazelli Coltro, in Capillary Electrophoresis and Microchips, 2013, pp. 145-160.

26 D. Connolly, P. Floris, B. Paull and P. N. Nesterenko, TrAC, Trends Anal. Chem., 2010, 29, 870-884.

27 Z. Walsh, P. a. Levkin, S. Abele, S. Scarmagnani, D. Heger, P. Klán, D. Diamond, B. Paull, F. Svec and M. Macka, J. Chromatogr. A, 2011, 1218, 2954-2962.

28 F. Opekar, P. Tůma and K. Štulík, Sensors, 2013, 13, 27862801.

29 X. Zhang, Q. Li, X. Jin, C. Jiang, Y. Lu, R. Tavallaie and J. J. Gooding, Sci. Rep., 2015, 5, 12539.

30 D. Connolly, L. P. Barron, E. Gillespie and B. Paull, Chromatographia, 2009, 70, 915-920.

31 E. Gillespie, D. Connolly and B. Paull, Analyst, 2009, 134, 1314-1321.

32 D. Connolly and B. Paull, J. Sep. Sci., 2009, 32, 2653-2658.

33 K. Mayrhofer, A. J. Zemann, E. Schnell and G. K. Bonn, Anal. Chem., 1999, 71, 3828-3833.

34 J. G. Alves Brito-Neto, J. A. Fracassi Da Silva, L. Blanes and C. L. Do Lago, Electroanalysis, 2005, 17, 1198-1206.

35 E. Baltussen and S. Baltussen, Electrophoresis, 2002, 23, 2888-2893.

36 J. G. Alves Brito-Neto, J. A. Fracassi Da Silva, L. Blanes and C. L. Do Lago, Electroanalysis, 2005, 17, 1207-1214.

37 P. Kubáň and P. C. Hauser, Electrophoresis, 2004, 25, 33983405.

38 A. J. Zemann, E. Schnell, D. Volgger and G. K. Bonn, Anal. Chem., 1998, 70, 563-567.

39 J. a. Fracassi Da Silva, N. Guzman and C. L. Do Lago, J. Chromatogr. A, 2002, 942, 249-258.

40 F. Tan and Y. Guan, Electrophoresis, 2005, 23, 152-157.

41 B. Gaš, J. Zuska, P. Coufal and T. Van De Goor, Electrophoresis, 2002, 23, 3520-3527.

42 M. Zhang, B. N. Stamos, N. Amornthammarong and P. K. Dasgupta, Anal. Chem., 2014, 86, 11538-11546.

43 J. A. F. da Silva and C. L. do Lago, Anal. Chem., 1998, 70, 4339-4343.

44 M. Stojkovic, B. Schlensky and P. C. Hauser, Electroanalysis, 2013, 25, 2645-2650.

45 P. Kubán and P. C. Hauser, Lab Chip, 2005, 5, 407-415.

46 Y. Ren, H. Deng, W. Shen and Z. Gao, Anal. Chem., 2013, 85, 4784-4789.

47 B. C. Beard, A. Johnson, F. M. Cambria and P. N. Trinh, J. Cosmet. Sci., 2005, 56, 65-77.

48 J. Liu, S. Fu, B. Yuan, Y. Li and Z. Deng, J. Am. Chem. Soc., 2010, 132, 7279-7281. 